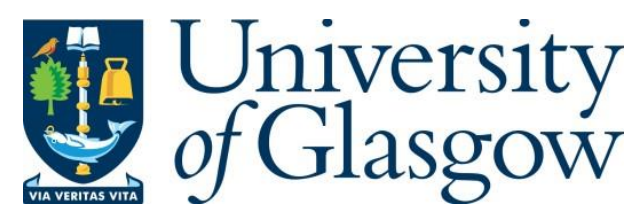

Overman, M. J. et al. (2017) Glycemia but not the metabolic syndrome is associated with cognitive decline: findings from the European Male Ageing Study. American Journal of Geriatric Psychiatry, 25(6), pp. 662-671.

There may be differences between this version and the published version. You are advised to consult the publisher's version if you wish to cite from it.

http://eprints.gla.ac.uk/138355/

Deposited on: 13 February 2018

Enlighten - Research publications by members of the University of Glasgow http://eprints.gla.ac.uk 


\section{Glycaemia but not the metabolic syndrome is associated with cognitive}

\section{3 decline: Findings from the European Male Ageing Study}

Margot J Overman ${ }^{1}$, M.Sc., Neil Pendleton ${ }^{2}$, M.D., Terence W O’Neill ${ }^{3,4}$, M.D., Gyorgy Bartfai ${ }^{5}$, M.D., Felipe F Casanueva ${ }^{6}$, M.D., B.Sc Gianni Forti ${ }^{7}$, M.D., Giulia Rastrelli ${ }^{8}$, M.D., Aleksander Giwercman ${ }^{9}$, M.D., Thang S Han ${ }^{10}$, Ph.D, Ilpo T Huhtaniemi ${ }^{11}$, M.D., Krzysztof Kula ${ }^{12}$, M.D., Michael EJ Lean ${ }^{13}$, M.D., Margus Punab ${ }^{14}$, M.D., David M Lee ${ }^{15}$, Ph.D., Elon S Correa ${ }^{16}$, Ph.D., Tomas Ahern ${ }^{16}$, M.D., Michaël R Laurent ${ }^{1,17}$, M.D., Sabine MP Verschueren ${ }^{18}$, Ph.D., Leen Antonio ${ }^{19,20}$, M.D., Evelien Gielen ${ }^{1,}$ ${ }^{21}$, M.D., Martin K Rutter ${ }^{22,23}$, M.D., Dirk Vanderschueren ${ }^{19}$, M.D., Frederick CW Wu ${ }^{16}$, M.D., Jos Tournoy ${ }^{1,21}$, M.D., the EMAS study group.

Author affiliations: ${ }^{1}$ Gerontology and Geriatrics, Department of Clinical and Experimental Medicine, KU Leuven, Leuven, Belgium; ${ }^{2}$ Clinical \& Cognitive Neurosciences, Institute of Brain, Behaviour and Mental Healthy, The University of Manchester, UK; ${ }^{3}$ Arthritis Research UK Centre for Epidemiology, Institute of Inflammation and Repair, the University of Manchester, Manchester, UK; ${ }^{4} \mathrm{NIHR}$ Manchester Musculoskeletal Biomedical Research Unit, Central Manchester University Hospitals NHS Foundation Trust, Manchester Academic Health Science Centre, Manchester, UK; ${ }^{5}$ Department of Obstetrics, Gynaecology and Andrology, Albert Szent-György Medical University, Szeged, Hungary; ${ }^{6}$ Department of Medicine, Instituto Salud Carlos III, Complejo Hospitalario Universitario de Santiago (CHUS); ${ }^{7}$ Endocrinology Unit, University of Florence, Florence, Italy; ${ }^{8}$ Sexual Medicine and Andrology Unit, Department of Experimental, Clinical, and Biomedical Sciences, University of Florence, Florence, Italy; ${ }^{9}$ Reproductive Medicine Centre, Skåne University Hospital, University of Lund, Lund, Sweden; ${ }^{10}$ Department of Endocrinology, Ashford and St Peter's NHS Foundation Trust, Chertsey, UK; ${ }^{11}$ Department of Surgery and Cancer, Imperial College London, Hammersmith Campus, London, UK; ${ }^{12}$ Department of Andrology and Reproductive Endocrinology, Medical University of Łódź, Łódź, Poland; ${ }^{13}$ Department of Human Nutrition, University of Glasgow, Glasgow, UK; ${ }^{14}$ Andrology Unit, United Laboratories of Tartu University Clinics, Tartu, Estonia; ${ }^{15}$ School of Social Sciences, Cathie 
Marsh Institute for Social Research, The University of Manchester, Manchester, UK; ${ }^{16}$ Andrology

Research Unit, The University of Manchester, Manchester, UK; ${ }^{17}$ Laboratory of Molecular Endocrinology, KU Leuven, Heverlee, Belgium; ${ }^{18}$ Department of Rehabilitation Sciences, KU Leuven, Leuven, Belgium; ${ }^{19}$ Department of Andrology and Endocrinology, KU Leuven, Leuven, Belgium; ${ }^{20}$ Department of Clinical and Experimental Medicine, KU Leuven, Leuven, Belgium; ${ }^{21}$ Geriatric Medicine, University Hospitals Leuven, Leuven, Belgium; ${ }^{22}$ The Endocrinology and Diabetes, Research Group, Faculty of Medical and Human Sciences, Institute of Human Development, University of Manchester, Manchester, UK; ${ }^{23}$ Manchester Diabetes Centre, Central Manchester University Hospitals NHS Foundation Trust, Manchester Academic Health Science Centre, Manchester, UK.

\section{Corresponding author: Margot Overman}

John Radcliffe Hospital

West Wing, Level 6

Oxford, OX3 9DU

Email address: margot.overman@ndcn.ox.ac.uk

Telephone number: +44 (0) 7759478612

Sources of support: This work was supported by the Commission of the European Communities Fifth Framework Program "Quality of Life and Management of Living Resources” Grant QLK6-CT-2001-00258. Additional support was provided by the Arthritis Research Campaign (UK).

Acknowledgments: The authors wish to thank the participating patients in the eight countries, and the following research and nursing staff for their data collection: C Pott, Manchester; E Wouters, Leuven; M Nilsson, Malmö; M del Mar Fernandez, Santiago de Compostela; M Jedrzejowska, Łódź; H-M Tabo, Tary; A Heredi, Szeged. The authors also wish to thank C 
52 Moseley, Manchester, for data entry and project co-ordination and E van Herck, Leuven, for performing the 25(OH)D assays. The EMAS Study Group members are based in the following cities: Florence, Italy (Gianni Forti, Luisa Petrone, Giovanni Corona); Leuven, Belgium (Dirk Vanderschueren, Herman Borghs, Leen Antonio); Łódź, Poland (Krzysztof Kula, Jolanta Slowikowska-Hilczer, Renata Walczak-Jedrzejowska); London, UK (Ilpo Huhtaniemi); Malmö, Sweden (Aleksander Giwercman); Manchester, UK (Frederick Wu, Alan Silman, Neil Pendleton, Terence O’Neill, Joseph Finn, Philip Steer, David Lee, Stephen Pye); Santiago de Compostela, Spain (Felipe Casanueva, Ana I Castro); Szeged, Hungary (Gyorgy Bartfai, Imre Földesi, Imre Fejes); Tartu, Estonia (Margus Punab, Paul Korrovitz).

No disclosures to report.

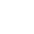

(1)

5

66

67

68

Key words: "Metabolic syndrome", “Cognition”, “Ageing”, "Male health", "Multicentre study" 
Objectives: Previous research has indicated that components of the metabolic syndrome (MetS), such as hyperglycaemia and hypertension, are negatively associated with cognition. However, evidence that MetS itself is related to cognitive performance has been inconsistent. In this longitudinal study, we aimed to investigate whether MetS or its components affect cognitive decline in ageing men and whether any interaction with inflammation existed.

Design: Longitudinal study over a mean of $4.4(\mathrm{SD} \pm 0.3)$ years.

Setting: Multi-centre European male Ageing Study (EMAS).

Participants: Men aged 40-79 years.

Measurements: Cognitive functioning was assessed using the Rey-Osterrieth Complex Figure (ROCF), the Camden Topographical Recognition Memory (CTRM) task, and the Digit Symbol Substitution Test (DSST). High-sensitivity C-reactive protein (hs-CRP) levels were measured using a chemiluminescent immunometric assay.

Results: Overall, 1,913 participants contributed data to the ROCF analyses and 1,965 subjects contributed to the CTRM and DSST analyses. In multiple regression models, the presence of baseline MetS was not associated with cognitive decline over time $(p>0.05)$. However, logistic ordinal regressions indicated that high glucose levels were related to a greater risk of decline on the ROCF Copy $(\beta=-0.42, p<0.05)$ and the DSST $(\beta=-0.39, p<0.001)$. There was neither a main effect of hs-CRP levels nor an interaction effect of hs-CRP and MetS at baseline on cognitive decline.

Conclusions: We found no evidence for a relationship between MetS or inflammation and cognitive decline in this sample of ageing men. However, glycaemia was negatively associated with visuoconstructional abilities and processing speed. 
The metabolic syndrome (MetS) is a cluster of cardiovascular risk factors which includes abdominal obesity, hypertension, hypertriglyceridemia, low high-density lipoprotein cholesterol (HDL-c), and elevated blood glucose levels[1], affecting 20-30\% of adults suffering from MetS worldwide[2]. MetS and its components are associated with a range of negative cardiovascular health outcomes[3]. Emerging evidence suggests that MetS may also present an increased risk of dementia[4-7] and accelerated decline of cognitive abilities such as visual working memory[8] and executive functioning[9,10]. However, while some studies indicate that MetS affects cognition over and above the sum of its individual components[11,12], not all research has confirmed a synergistic effect of MetS criteria on cognitive decline. The longitudinal Atherosclerosis Risk in Communities Study found that hypertension and diabetes mellitus were associated with greater declines in processing speed, verbal memory, and executive functioning in middle-aged men, whereas MetS as a whole was not[13]. Similarly, prospective data of 2,476 older adults indicated that risk factors such as hyperinsulinemia and diabetes but not MetS were associated with an increased risk of developing dementia[14]. Moreover, a large multi-centre study of 7,087 community-dwelling older adults suggested that MetS does not predict development of dementia any better than its' separate components[15]. It is therefore not yet clear whether MetS is a better predictor of cognitive ageing than its individual components. The relationship between MetS and cognition may be further complicated by concurrent inflammatory processes, with the first increasing the latter and vice versa[11]. In people with MetS, some cross-sectional and longitudinal studies have primarily observed poor cognitive performance[16-18] and mild cognitive impairment[19] in individuals who present with high serum markers of inflammation. The inflammatory response should therefore be considered when examining the association between MetS and cognitive ageing. We present longitudinal findings from the European Male Ageing Study (EMAS), a multi-centre cohort study of middle-aged and older men[20]. The main objective was to investigate whether baseline MetS was associated with cognitive decline over time in ageing men. Furthermore, we aimed to examine the impact of individual MetS components on cognitive ageing. Lastly, we explored potential interaction effects of MetS and hs-CRP levels, a biomarker of inflammation, on the rate of cognitive decline. 


\section{METHODS}

\section{Study participants}

130

131

132

133

134

135

Recruitment and assessment of participants of the European Male Ageing Study have been described in detail elsewhere[20]. Briefly, 3,369 community-dwelling men aged 40 to 79 years were recruited from population and health registers in centres based in Leuven, Belgium; Manchester, UK; Florence, Italy; Łódź, Poland; Malmö, Sweden; Santiago de Compostela, Spain; Szeged, Hungary; and Tartu, Estonia. A letter of invitation including a postal questionnaire was sent to gather information about education, general health, and physical activity. The mean adjusted response rate across the eight centres was $43 \%$. Men who agreed to partake were invited to attend for physical and cognitive performance measures, an interviewer-assisted questionnaire, and a fasting blood test. Follow-up assessments took place at a mean of $4.4(\mathrm{SD} \pm 0.3)$ years after the baseline measurements. Ethical approval was obtained in accordance with local practice and institutional requirements in each centre. All participants gave their written informed consent.

\section{Interviewer-assisted questionnaire and anthropometry}

The interviewer-assisted questionnaire included the Beck Depression Inventory-II (BDI) for subjective depressive symptoms[21], the Physical Activity Scale for the Elderly (PASE)[22], and questions regarding smoking habits, alcohol consumption, and health. Height and weight were measured using standard, calibrated instruments[20]. Current prescription and non-prescription medication use was selfreported by the participants.

\section{Cardiovascular risk factors}

Seated pulse and blood pressure was recorded following a rest period of 5 minutes using an automated sphygmomanometer (Omron 500I, Omron Healthcare (UK), Ltd Milton Keynes, UK). Waist circumference was measured three times using anthropometric tape, with the median value being used for analyses. Morning phlebotomy was performed before 10am to obtain a fasting blood sample. Analyses of triglyceride and HDL-c levels were performed in local centres with commercially available enzymatic assays. The presence of metabolic syndrome was determined according to the National Cholesterol Education Program Adult Treatment Panel-III (ATP-III) definition[1]. Participants were 
classified as having MetS if three or more of the following criteria were met: waist circumference $>102$ $\mathrm{cm}$, fasting triglyceride $>1.7 \mathrm{mmol} / \mathrm{l}$, fasting HDL-c $<1.03 \mathrm{mmol} / \mathrm{l}$, blood pressure $>130 / 85 \mathrm{mmHg}$ or current use of anti-hypertensive medication, and fasting glucose $>5.6 \mathrm{mmol} / \mathrm{l}$ or current use of antidiabetic medication. Levels of hs-CRP were measured using a solid-phase chemiluminescent immunometric assay (Immulite 2000 hs-CRP assay; Diagnostics Products Corporation, Siemens, Deerfield, IL, USA) with a sensitivity of $0.1 \mathrm{~g} / \mathrm{l}$. The intra- and inter-assay coefficients of variation were $2.8 \%$ and $3.1 \%$, respectively.

\section{Cognitive performance}

The EMAS cognitive test battery consisted of four tasks: the Rey-Osterrieth Complex Figure (ROCF) Copy and Recall, the Camden Topographical Recognition Memory (CTRM) task, and the Digit Symbol Substitution Test (DSST). The ROCF tasks provide an indication of visuo-constructional abilities and memory recall[23]. In the Copy component, participants were instructed to copy an abstract figure as accurately as possible within a 5-minute time limit. In the Recall task, participants were asked without previous warning to draw the figure from memory thirty minutes after completing the copy. Scoring criteria were based on the original procedure, with a maximum score of 36 points. The CTRM assesses visual recognition memory[24] and involves the sequential presentation of photographs of urban scenes followed by a forced-choice recognition component. One point was given for each correctly identified image, with a maximum score of 30. Finally, the DSST is a paper-and-pencil subtest from the Wechsler Adult Intelligence Scale used to measure psychomotor speed and visual scanning[25]. Participants had to substitute as many symbols for digits as possible within 60 seconds using a coding table.

\section{Statistical analysis}

Participants with incomplete cognitive or MetS data at baseline or follow-up were excluded from the analyses. Characteristics of the study sample at baseline were compared by MetS status using MannWhitney $U$ tests for continuous variables and $\chi^{2}$ tests for dichotomous variables. Continuous cognitive change was calculated by subtracting the baseline score from the follow-up score. In order to specifically compare participants with significant cognitive decline to those who did not demonstrate cognitive decline, cognitive change was also investigated as a categorical variable. Participants were divided into 
the categories "Decline" ( $>1$ SD decrease from baseline), "No change" ( $<1$ SD change from baseline), and "Improvement" (>1 SD increase from baseline). Furthermore, we examined the effect of persistent MetS over time, defined as the presence of MetS at both baseline and follow-up measurements. Dichotomous variables (absent vs. present) were created for baseline MetS status as well as for the individual MetS criteria based on the ATP-III definition.

Age-adjusted linear regressions were performed to examine the relationship between continuous cognitive decline and MetS. Categorical cognitive decline was investigated using age-adjusted ordinal logistic regressions. Predictors were baseline MetS status, number of MetS criteria present (0-5), and individual dichotomised MetS criteria. Subsequently, further adjustments were made for education (years), BDI score, smoking (non-smoker vs. currently smoking), alcohol consumption $(<1$ day/week vs. $\geq 1$ day/week), PASE score, and centre. Finally, analyses were adjusted for the presence of heart disease and stroke. An interaction term between BDI score and baseline MetS was included to assess potential moderation effects of depressive symptoms on the relationship between MetS and cognitive decline, as previous research has indicated that MetS is associated with an increased risk of depression[26] which can negatively affect cognition[27]. Furthermore, an interaction term between hsCRP and baseline MetS status was used as a predictor variable to examine the effect of inflammation on MetS and cognitive decline. Results are expressed as unstandardized beta coefficients $(\beta)$ and 95\% confidence intervals. Statistical analyses were undertaken using Stata version 13.1 (StataCorp, College Station, TX, USA).

\section{RESULTS}

\section{Cohort characteristics}

Of the men who participated in baseline assessments, 2,738 (86.1\% of survivors) returned for followup measurements. A total of 698 participants was excluded from the analyses due to incomplete MetS data, resulting in a final cohort of 1,913 participants for the ROCF tests and 1,965 participants for the CTRM and DSST. Baseline characteristics of the sample by MetS status are shown in Table 1. On average, participants with MetS at baseline were older, showed more subjective depressive symptoms, were less physically active, had higher levels of hs-CRP, consumed less alcohol, and were more likely 
to have a history of heart disease or diabetes. The prevalence of MetS was $28.8 \%$ at baseline and $32.6 \%$ at follow-up. A $\chi^{2}$ test revealed that baseline MetS incidence differed significantly by centre $(p<0.001)$, ranging from $18.3 \%$ in Leuven to $44.2 \%$ in Szeged. The most common MetS criterion met was hypertension (85\%), followed by abdominal obesity (35\%), hyperglycaemia (33\%), hypertriglyceridaemia (28\%), and high HDL-c levels (13\%). Mean cognitive scores on the ROCF Recall and CTRM tasks improved slightly over time, while a decline was observed for the DSST (see Table 2). A decline of 1SD or more from baseline occurred in $9.0 \%$ of participants on the ROCF Copy, $16.0 \%$ on the ROCF Recall, $15.8 \%$ on the CTRM, and $20.8 \%$ on the DSST. Mann-Whitney tests showed that participants who were lost to follow-up had lower baseline scores on the ROCF Copy $(U=-9.01, p$ $<0.001)$, ROCF Recall $(U=-8.87, p<0.001), \operatorname{CTRM}(U=6.85, p<0.001)$, and DSST $(U=-11.21, p$ $<0.001)$ than those who returned for follow-up. Furthermore, a $\chi^{2}$ test indicated that participants with MetS at baseline were more likely to be lost to follow-up than those who did not have MetS $(p<0.001)$.

\section{Metabolic syndrome and cognitive decline}

Linear regression models of baseline MetS status, MetS components, and continuous cognitive decline are summarised in Table 3. In age-adjusted models, MetS, large waist circumference, and high blood pressure were related to a better performance on the ROCF Recall. Furthermore, hypertriglyceridemia was associated with a greater decline on the DSST. None of the associations were maintained after adjusting for confounders such as education, physical activity, and centre. Furthermore, interaction terms between age or BDI score and MetS or MetS components were not significant for any of the cognitive tasks (all $p>0.05$ ). An increasing number of MetS components was related to better ROCF and poorer DSST performance in age-adjusted but not fully-adjusted models (see Table 3).

When cognitive scores were investigated as categorical variables, there was a significant negative association between baseline MetS and performance on the ROCF Copy and DSST in ageadjusted models (see Table 4). In addition, an increasing number of MetS components was related to a worsening performance on the ROCF Copy, the CTRM, and the DSST. Of the individual components, large waist circumference was related to poor performance on the DSST, high glucose levels correlated with decline on the ROCF Copy, CTRM, and DSST, and high HDL-c was associated with decline on 
the ROCF Recall. After adjusting for additional confounders, logistic regressions indicated that the relationship between glucose levels and decline on the ROCF Copy and DSST remained significant. When these associations were analysed separately for participants $<65$ years and participants $\geq 65$ years at baseline, only the correlation between ROCF Copy change remained significant for older $(\beta=-0.74$, $p<0.01)$ but not younger participants $(\beta=-0.19, p=0.541)$. Finally, persistent MetS was associated with greater decline on the DSST in age-adjusted $(\beta=-1.02, p<0.01)$ but not fully adjusted models $(\beta$ $=-0.81, p=0.225)$. There were no significant associations between persistent MetS and decline on the ROCF or CTRM tasks (data not shown).

\section{Cognitive decline and hs-CRP}

Baseline hs-CRP levels were not independently associated with decline on any of the four tasks in either age- or fully-adjusted linear regression models (all $p>0.05$ ). Furthermore, there were no significant interaction effects of hs-CRP and MetS on cognitive performance on either the ROCF Copy $(\beta=-0.37$, $p=0.220)$, the ROCF Recall $(\beta=-0.32, p-0.501)$, the CTRM $(\beta=-0.32, p=0.317)$, or the DSST $(\beta=$ $0.12, p=0.750)$.

\section{CONCLUSIONS}

In this cohort of ageing European men, we found no evidence for a longitudinal association between baseline MetS status or the cumulative effect of its components and cognitive decline over a mean period of 4.4 years. However, hyperglycaemia was associated with an increased risk of decline in visuoconstructional abilities and processing speed. These results are consistent with cross-sectional findings from the EMAS study that glucose level but not MetS was related to cognitive performance[28]. In accordance with the present findings, several prospective studies reported no significant correlations in either middle-aged[13] or older populations[15,29,30]. Although a number of longitudinal studies found a correlation between MetS and memory[8,10], executive function[10,31], and processing speed[17,30], effect sizes are generally small[32]. Disparate outcomes in prior studies may in part be caused by differences in methodology and samples. A meta-analysis of 13 longitudinal studies found that, across investigations, 17 different tasks were employed to assess cognitive functioning[32], complicating direct comparisons. In addition, several studies opted for an alternative definition instead of the commonly 
used ATP-III guidelines. However, longitudinal studies using the World Health Organisation[33] or American Heart Association[34] guidelines also reported non-significant associations between MetS and cognition. Nevertheless, use of alternative diagnostic criteria might yield different results. Finally, it has been suggested that MetS affects cognition more strongly in women than in men[5,10,15,35,36], although the reverse pattern has also been observed[18]. Genetic dissimilarities may make women more vulnerable to the influence of vascular risk factor on the brain than men[15], which could explain the null findings in our all-male cohort. However, little is known about possible biomechanisms which could account for a gender-dependent association between MetS and cognition and additional research is needed.

While there is thus no conclusive evidence for a relationship between MetS and cognitive ageing, prior research has frequently reported associations between the individual MetS components and cognition. While the majority of studies indicates that hyperglycaemia or diabetes[18,20,33,37] and hypertension $[13,18,33,37]$ in particular are detrimental to cognitive functions, others suggest that HDLglucose levels correlating with declines in visuoconstructional abilities and processing speed. Therefore, hyperglycaemia and/or diabetes may be driving associations between MetS and cognitive decline. Possible mechanisms by which hyperglycaemia could affect cognitive functions include increases in early pre-programmed cell death[37] and microvascular disease[38]. Although we found that waist circumference, blood pressure, and hypertriglyceridemia were also related to ROCF Recall and DSST performance, these associations were mainly explained by confounding factors. Previous studies which did not correct for the influence of these confounders may have overestimated the effect of these risk factors on cognition. Variance in age of the participants from different studies may also explain some of the conflicting findings, as it has been proposed that the influence of MetS components changes with age[39]. The present findings suggested that high glucose levels may be particularly detrimental to cognitive functioning in individuals aged 65 and over. However, we found no other age-related effects of MetS components on cognition in any of our regression models when including an interaction term between age and the individual components. Nevertheless, as our sample was relatively young, our 
findings may not be comparable to those studies investigating people aged 85 years and over.

Finally, some studies have indicated that the relationship between MetS and cognition is modified by inflammation, with the combined presence of MetS and high hs-CRP levels being associated with greater declines in global cognition[11,18,40] and non-amnestic mild cognitive impairment[19]. However, we found no evidence of an association between hs-CRP levels, MetS, and cognitive decline. Once again, this may be due to differences in population characteristics and cognitive assessments. For example, the longitudinal Sacramento Area Latino Study of Aging (SALSA), which found that high CRP levels were associated with greater cognitive decline in older adults with MetS than low CRP levels, used measures of global cognition and verbal abilities to examine cognitive decline[41]. It is possible that the cognitive tasks used in the present study, which focused on visuospatial functioning and processing speed, were unable to capture potential interaction effects of MetS and inflammation. In addition, studies investigating the relationship between CRP, the metabolic syndrome, and cognition tend to define high and low inflammation based on distributions within the participant sample $[16,42]$ rather than using a pre-specified value. A wide variety of values has therefore been used to define 'high' inflammation, making it difficult to establish how CRP levels relate to MetS and cognition. Other biomarkers such as interleukin-6 (IL-6), homocysteine, and $\alpha 1$-antichymotrypsin may be more strongly correlated with cognitive decline[43]. Alternatively, some studies have used a combination of inflammatory markers, such as CRP and IL-6 measures, to define high inflammation rather than investigating the biomarkers in isolation[16]. Future research including or combining other inflammation biomarkers may contribute to our understanding of the role of inflammation in the relationship between MetS and cognition.

Major strengths of EMAS are its prospective and multi-centre design and the broad range of physiological and performance measures collected. One limitation is that our assessment of cognitive domains was constrained by the necessary use of culture- and language-fair instruments. We therefore cannot draw conclusions about the effects of MetS on semantic abilities in men. In addition, performance on the ROCF and CTRM tasks may be influenced by a practice effect, as underscored by an average improvement in scores over time, resulting in an underestimation of cognitive decline in our cohort. However, when we investigated the participants with the greatest cognitive decline in a sub-analysis, 
we also did not find a significant association with MetS. As we did not have information about the presence of MetS prior to our baseline measurements, we were not able to investigate the influence of

321

322

323 any long-term duration of MetS on cognition. However, we found no association between persistent MetS over 4.4 years and cognitive function. We cannot exclude that a longer duration could be an important factor in predicting cognitive decline, as a study of middle-aged adults indicated that MetS was only related to decline in verbal abilities if the syndrome persisted over 10 years[44]. However, the Caerphilly Prospective Study also found no association between length of MetS exposure and cognitive performance in a group of middle-aged men over 14 years[35]. It is therefore not yet clear whether the duration of MetS is related to cognitive decline. As we conducted multiple tests to investigate the relationship between metabolic syndrome and cognitive decline, there was an increased risk of Type I errors. Although the results therefore need to be interpreted with caution, the absence of an association between MetS and cognition in any of our analyses reinforces our conclusion that the syndrome may be unrelated to cognitive decline. Another limitation to our study is that participants lost to follow-up on average had lower cognitive scores and were more likely to have MetS. It is therefore possible that individuals with MetS showing the greatest cognitive impairments were not included in this study. Moreover, we acknowledge that a relatively small number of participants showed significant cognitive decline on several of the tasks used. Although we cannot exclude the possibility that the present study lacked power to investigate associations with cognitive decline, the significant relationships between cognition and hyperglycaemia suggest that this is not the case. Moreover, several larger prospective studies have similarly failed to find a relationship between MetS and cognition, providing further support for our results[13,15,16]. It should be noted that our results are based on a relatively healthy cohort of European men. As significant associations between MetS and cognitive decline have previously been reported in other ethnic groups, including Latino [41], Chinese [45], and Korean participants [46], the present findings should be extrapolated to other populations with care. In addition, as we did not include measures of mild cognitive impairment or dementia, it is unclear whether the metabolic syndrome might be associated with more severe cognitive impairments. Finally, although we adjusted for the influence of alternative factors which may be associated with cognitive decline and dementia independent of MetS, there are a number of potential predictors which were not investigated 
here. For example, risk factors and biomarkers such as the APOE4 allele[47], amyloid load[48], and hippocampal volume[49] have been related to cognitive decline and risk of dementia in healthy older adults. We cannot exclude the possibility that factors such as these affected our results. Further research is needed to clarify the relationship between MetS and dementia syndromes.

\section{CONCLUSION}

In view of the large percentage of the population affected by metabolic syndrome, it is important to understand the consequences of the MetS on general health. Although some previous research suggests that MetS negatively affects cognition over and above individual cardiovascular risk factors, we did not find evidence that MetS is related to cognitive decline with age in a large sample of middle-aged and older European men. Additionally, there was no indication that inflammatory processes worsened cognitive performance. However, our findings indicate that hyperglycaemia may have a significant negative effect on several domains of cognitive decline with age. Further research is needed to explore whether the findings from the EMAS cohort extend to other populations.

\section{REFERENCES}

1. Grundy SM, Brewer Jr HB, Cleeman JI, et al: Definition of metabolic syndrome: Report of the National Heart, Lung, and Blood Institute/American Heart Association Conference on Scientific Issues Related to Definition. Circulation 2004; 109:433-438.

2. Grundy SM: Metabolic syndrome pandemic. Arterioscler Thromb Vasc Biol 2008; 28:629-636.

3. Isomaa B, Almgren P, Tuomi T, et al: Cardiovascular morbidity and mortality associated with the metabolic syndrome. Diabetes Care 2001; 24:683-689.

4. Kalmijn S, Foley D, White L, et al: Metabolic cardiovascular syndrome and risk of dementia in Japanese-American elderly men: The Honolulu-Asia Aging Study. Arterioscl Thromb Vasc Biol 2000; 20:2255-2260.

5. Vanhanen M, Koivisto K, Moilanen L, et al: Association of metabolic syndrome with Alzheimer disease: A population-based study. Neurology 2006; 67:843-847. 
6. Ng TP, Feng L, Nyunt M, et al: Metabolic syndrome and the risk of mild cognitive impairment and progression to dementia: Follow-up of the Singapore Longitudinal Ageing Study Cohort. JAMA Neurol 2016; 73:456-463.

7. Solfrizzi V, Scafato E, Capurso C, et al: Metabolic syndrome and the risk of vascular dementia: The Italian Longitudinal Study on Ageing. J Neurol Neurosurg Psychiatry 2010; 81:433-440.

8. Raffaitin C, Féart C, Le Goff M, et al: Metabolic syndrome and cognitive decline in French elders: The Three-City Study. Neurology 2011; 76:518-525.

9. McEvoy LK, Laughlin GA, Barrett-Connor E, et al: Metabolic syndrome and 16-year cognitive decline in community-dwelling older adults. Ann Epidemiol 2012; 22:310-317.

10. Levin BE, Llabre MM, Dong C, et al: Modeling metabolic syndrome and its association with cognition: The Northern Manhattan Study. J Int Neuropsychol Soc 2014; 20:951-960.

11. Yaffe K: Metabolic syndrome and cognitive disorders: Is the sum greater than its parts? Alzheimer Dis Assoc Disord 2007; 21:167-171.

12. Hao Z, Wu B, Wang D \& Liu M: Association between metabolic syndrome and cognitive decline: A systematic review of prospective population-based studies. Acta Neuropsychiatr 2011; 23:69-74.

13. Dearborn JL, Knopman D, Sharrett AR, et al: The metabolic syndrome and cognitive decline in the Atherosclerosis Risk in Communities Study (ARIC). Dement Geriatr Cogn Disord 2014; $38: 337-346$

14. Muller M, Tang M, Schupf N, et al: Metabolic syndrome and dementia risk in a multiethnic elderly cohort. Dement Geriatr Cogn Disord 2007; 24:185-192.

15. Raffaitin C, Gin H, Empana J, et al: Metabolic syndrome and risk for incident Alzheimer's disease or vascular dementia: The Three-City Study. Diabetes Care 2009;32:169-174.

16. Yaffe K, Kanaya A, Lindquist K, et al: The metabolic syndrome, inflammation, and risk of cognitive decline. JAMA 2004; 292:2237-2242.

17. Dik MG, Jonker C, Comijs HC, et al: Contribution of metabolic syndrome components to cognition in older individuals. Diabetes Care 2007; 30:2655-2660. 
18. Cavalieri M, Ropele S, Petrovic K, et al: Metabolic syndrome, brain magnetic resonance imaging, and cognition. Diabetes Care 2010; 33:2489-2495.

19. Roberts RO, Geda YE, Knopman DS, et al: Metabolic syndrome, inflammation, and nonamnestic mild cognitive impairment in older persons: A population-based study. Alzheimer Dis Assoc Disord 2010; 24:11-18.

20. Lee DM, O’Neill TW, Pye SR, et al: The European Male Ageing Study (EMAS): Design, methods and recruitment. Int J Androl 2009; 32:11-24.

21. Beck AT, Steer RA, Brown GK: Manual for the Beck Depression Inventory-II. San Antonio, TX, USA: Psychological Corporation, 1996.

22. Washburn RA, Smith KW, Jette AM, et al: The Physical Activity Scale for the Elderly (PASE): Development and evaluation. J Clin Epidemiol 1993; 46:153-162.

23. Osterrieth PA: Le test de copie d'une figure complexe. Arch Psychol 1944; 30:206-356.

24. Warrington EK: The Camden memory tests manual (Vol. 1.): Psychology Press, 1996.

25. Uiterwijk JM: WAIS-III-NL-V. Lisse, The Netherlands: Swets \& Zeitlinger, 2001.

26. Koponen H, Jokelainen J, Keinänen-Kiukaanniemi S, et al: Metabolic syndrome predisposes to depressive symptoms: A population-based 7-year follow-up study. J Clin Psychiatry 2008; 69:178-182.

27. Rock PL, Roiser JP, Riedel WJ, et al: Cognitive impairment in depression: A systematic review and meta-analysis. Psychol Med 2014;44:2029-2040.

28. Tournoy J, Lee DM, Pendleton N, et al: Association of cognitive performance with the metabolic syndrome and with glycaemia in middle-aged and older European men: The European Male Ageing Study. Diabetes Metab Res Rev 2010; 26:668-676.

29. Harrison SL, Stephan BCM, Siervo M, et al: Is there an association between metabolic syndrome and cognitive function in very old adults? The Newcastle $85+$ Study. J Am Geriatr Soc 2015; 63:667-675.

30. Katsumata Y, Todoriki H, Higashiuesato Y, et al: Metabolic syndrome and cognitive decline among the oldest old in Okinawa: In search of a mechanism. The KOCOA Project. J Gerontol A Biol Sci Med Sci 2012; 67A:126-134. 
31. Reijmer YD, Van den Berg E, Dekker JM, et al: The metabolic syndrome, atherosclerosis and cognitive functioning in a non-demented population: The Hoorn Study. Atherosclerosis 2011; 219:839-845.

32. Siervo M, Harrison SL, Jagger C, et al: Metabolic syndrome and longitudinal changes in cognitive function: A systematic review and meta-analysis. J Alzheimers Dis 2014; 41:151-161.

33. Laudisio A, Marzetti E, Pagano F, et al: Association of metabolic syndrome with cognitive function: The role of sex and age. Clin Nutr 2008; 27:747-754.

34. Schuur M, Henneman P, Van Swieten JC, et al: Insulin-resistance and metabolic syndrome are related to executive function in women in a large family-based study. Eur J Epidemiol 2010; 25:561-568

35. Creavin ST, Gallacher J, Bayer A, et al: Metabolic syndrome, diabetes, poor cognition, and dementia in the Caerphilly Prospective Study. J Alzheimers Dis 2012; 28:931-939.

36. Kesse-Guyot E, Julia C, Andreeva V, et al: Evidence of a cumulative effect of cardiometabolic disorders at midlife and subsequent cognitive function. Age Ageing 2015; 0:1-7.

37. Weinberger J, Biscarra V, Weisberg MK, et al: Factors contributing to strokes in patients with atherosclerotic disease of the great vessels: The role of diabetes. Stroke 1983; 14:709-712.

38. Baird TA, Parsons MW, Barber PA, et al: The influence of diabetes mellitus and hyperglycaemia on stroke incidence and outcome. J Clin Neurosci 2002; 9:618-626.

39. Panza F, Solfrizzi V, Logroscino G, et al: Current epidemiological approaches to the metaboliccognitive syndrome. J Alzheimers Dis 2012; 30:S31-S75.

40. Haffner SM: The metabolic syndrome: Inflammation, diabetes mellitus, and cardiovascular disease. Am J Cardiol 2006; 97:3A-11A.

41. Yaffe K, Haan M, Blackwell T, et al: Metabolic syndrome and cognitive decline in elderly Latinos: Findings from the Sacramento Area Latino Study of Aging study. J Am Geriatr Soc 2007: 55;758-762.

42. Cavalieri M, Ropele S, Petrovic K, et al: Metabolic syndrome, brain magnetic resonance imaging, and cognition. Diabetes Care 2010; 33: 2489 - 2495. 
43. Dik MG, Jonker C, Hack CE, et al: Serum inflammatory proteins and cognitive in decline in older persons. Neurology 2005; 64:1371-1377.

44. Akbaraly TN, Kivimaki M, Shipley MJ, et al: Metabolic syndrome over 10 years and cognitive functioning in late midlife: The Whitehall II study. Diabetes Care 2010; 33:84-89.

45. Ho RCM, Niti M, Yap KB, et al: Metabolic and cognitive decline in Chinese older adults: Results from the Singapore longitudinal ageing studies. Am J Geriatr Psychiatry 2008; 16:519522.

46. Lee KS, Eom JS, Cheong HK, et al: Effects of head circumference and metabolic syndrome on cognitive decline. Gerontology 2009; 56:32-38.

47. Tilvis RS, Kähönen-Väre M, Kolkkonen J, et al: Predictors of cognitive decline and mortality of aged people over a 10-year period. J Gerontol A Biol Sci Med Sci 2004; 59:M268-M274.

48. Lim YY, Ellis KA, Pietrzak RH, et al: Stronger effects of amyloid load than APOE genotype on cognitive decline in healthy older adults. Neurology 2012; 79:1645-1652. healthy elderly persons. JAMA Neurology 2002; 59:601-606. 
Table 1 Baseline characteristics of the EMAS cohort by baseline MetS status $(\mathrm{N}=1,965)$

\begin{tabular}{|c|c|c|c|}
\hline & $\begin{array}{r}\text { No MetS } \\
(\mathrm{n}=1,399 \\
71.2 \%)\end{array}$ & $\begin{array}{r}\text { MetS } \\
(\mathrm{n}=566 \\
28.8 \%)\end{array}$ & $p$-value \\
\hline \multicolumn{4}{|c|}{ Mean (SD) or \% } \\
\hline Age (years) & $58.5(10.7)$ & $60.0(10.3)$ & 0.005 \\
\hline $\begin{array}{l}\text { Age left education } \\
\text { (years) }\end{array}$ & $21.4(7.1)$ & $21.9(8.2)$ & 0.915 \\
\hline BDI score & $6.2(6.0)$ & $7.2(6.2)$ & $<0.001$ \\
\hline PASE score & $212.3(86.8)$ & $198.6(92.0)$ & 0.002 \\
\hline $\begin{array}{l}\text { Waist circumference } \\
\text { (cm) }\end{array}$ & $94.4(9.0)$ & $107.0(9.3)$ & $<0.001$ \\
\hline Triglycerides (mmol/l) & $1.2(0.6)$ & $2.0(0.8)$ & $<0.001$ \\
\hline Glucose (mmol/l) & $5.3(0.9)$ & $6.3(1.5)$ & $<0.001$ \\
\hline HDL-c (mmol/l) & $1.5(0.3)$ & $1.2(0.3)$ & $<0.001$ \\
\hline $\begin{array}{l}\text { Systolic blood } \\
\text { pressure (mmHg) }\end{array}$ & $143.3(20.2)$ & $151.4(19.4)$ & $<0.001$ \\
\hline $\begin{array}{l}\text { Diastolic blood } \\
\text { pressure (mmHg) }\end{array}$ & $86.0(11.7)$ & $90.6(11.8)$ & $<0.001$ \\
\hline Hs-CRP (mg/l) & $0.3(0.7)$ & $0.5(0.7)$ & $<0.001$ \\
\hline Current smoker (\%) & 19.6 & 20.1 & 0.208 \\
\hline $\begin{array}{l}\text { Alcohol consumption } \\
\geq 1 \text { day/week }(\%)\end{array}$ & 59.3 & 54.3 & 0.044 \\
\hline Heart condition (\%) & 14.0 & 18.4 & 0.017 \\
\hline Diabetes (\%) & 2.8 & 14.6 & $<0.001$ \\
\hline Stroke (\%) & 2.5 & 4.1 & 0.064 \\
\hline
\end{tabular}


2 Abbreviations: BDI, Beck Depression Inventory; PASE, Physical Activity Scale for the Elderly; hs3 CRP, high-sensitivity C-reactive protein. 
1 Table 2 Paired $t$-tests comparing mean (SD) cognitive performance scores at baseline and follow-up in 2 EMAS

\begin{tabular}{lrccr}
\hline & N & Baseline & Follow-up & $p$-value \\
\hline ROCF Copy & 1,913 & $34.0(3.8)$ & $34.0(4.0)$ & 0.778 \\
ROCF Recall & 1,913 & $17.8(6.4)$ & $18.5(7.0)$ & $<0.001$ \\
CTRM & 1,965 & $23.2(4.5)$ & $23.5(4.7)$ & 0.002 \\
DSST & 1,965 & $29.1(8.3)$ & $28.2(8.9)$ & $<0.001$
\end{tabular}

3 Abbreviations: ROCF, Rey-Osterrieth Complex Figure; CTRM, Camden Topographical Recognition 4 Memory; DSST, Digit Symbol Substitution Test.

5

6 
Table $3 \beta$-coefficients (95\% CI) of linear regressions for baseline MetS, number of MetS components, individual criteria, and continuous cognitive decline in EMAS

\begin{tabular}{|c|c|c|c|c|}
\hline & ROCF Copy & ROCF Recall & CTRM & DSST \\
\hline \multicolumn{5}{|l|}{ Model 1 ${ }^{a}$} \\
\hline Metabolic syndrome & $0.13(-0.23 ; 0.50)$ & $0.67(0.10 ; 1.24)^{*}$ & $0.06(-0.32 ; 0.44)$ & $-0.29(-0.74 ; 0.17)$ \\
\hline Number of MetS components $(0-5)$ & $0.07(-0.07 ; 0.21)$ & $0.32(0.09 ; 0.54)^{* *}$ & $0.04(-0.11 ; 0.18)$ & $-0.23(-0.41 ;-0.05)^{*}$ \\
\hline \multicolumn{5}{|l|}{ Individual criteria } \\
\hline Waist circumference $>102 \mathrm{~cm}$ & $0.08(-0.27 ; 0.43)$ & $0.61(0.06 ; 1.16)^{*}$ & $-0.18(-0.54 ; 0.18)$ & $-0.16(-0.60 ; 0.28)$ \\
\hline Blood pressure >130/85 and/or using anti- & $0.13(-0.34 ; 0.60)$ & $0.98(0.24 ; 1.73)^{*}$ & $-0.00(-0.49 ; 0.48)$ & $-0.29(-0.89 ; 0.31)$ \\
\hline \multicolumn{5}{|l|}{ hypertensive medication } \\
\hline Blood glucose $>5.6 \mathrm{mmol} / \mathrm{l}$ and/or using anti- & $0.28(-0.08 ; 0.63)$ & $0.22(-0.34 ; 0.78)$ & $0.17(-0.19 ; 0.53)$ & $0.39(-0.83 ; 0.06)$ \\
\hline \multicolumn{5}{|l|}{ diabetic medication } \\
\hline HDL-c $<1.03 \mathrm{mmol} / \mathrm{l}$ & $0.02(-0.47 ; 0.50)$ & $0.27(-0.50 ; 1.04)$ & $0.35(-0.16 ; 0.85)$ & $-0.38(-1.01 ; 0.24)$ \\
\hline Triglycerides > 1.5 mmol/l & $-0.03(-0.39 ; 0.34)$ & $0.45(-0.13 ; 1.03)$ & $0.08(-0.30 ; 0.46)$ & $-0.54(-1.01 ;-0.08)^{*}$ \\
\hline \multicolumn{5}{|l|}{ Model $2^{b}$} \\
\hline Metabolic syndrome & $0.05(-0.33 ; 0.44)$ & $0.48(-0.12 ; 1.07)$ & $0.24(-0.16 ; 0.64)$ & $0.04(-0.43 ; 0.52)$ \\
\hline Number of MetS components $(0-5)$ & $0.05(-0.10 ; 0.21)$ & $0.16(-0.07 ; 0.40)$ & $0.12(-0.03 ; 0.28)$ & $-0.07(-0.26 ; 0.12)$ \\
\hline
\end{tabular}




\begin{tabular}{|c|c|c|c|c|}
\hline \multicolumn{5}{|l|}{ Individual criteria } \\
\hline Waist circumference $>102 \mathrm{~cm}$ & $0.06(-0.31 ; 0.42)$ & $0.55(-0.01 ; 1.12)$ & $-0.01(-0.39 ; 0.37)$ & $0.03(-0.43 ; 0.49)$ \\
\hline $\begin{array}{l}\text { Blood pressure }>130 / 85 \text { and/or using anti- } \\
\text { hypertensive medication }\end{array}$ & $0.17(-0.33 ; 0.67)$ & $0.28(-0.49 ; 1.06)$ & $0.12(-0.40 ; 0.64)$ & $-0.23(-0.85 ; 0.39)$ \\
\hline $\begin{array}{l}\text { Blood glucose }>5.6 \mathrm{mmol} / \mathrm{l} \text { and/or using anti- } \\
\text { diabetic medication }\end{array}$ & $0.36(-0.02 ; 0.73)$ & $0.08(-0.50 ; 0.66)$ & $0.28(-0.11 ; 0.67)$ & $0.15(-0.31 ; 0.62)$ \\
\hline HDL-c $<1.03 \mathrm{mmol} / \mathrm{l}$ & $-0.24(-0.77 ; 0.29)$ & $0.21(-0.60 ; 1.03)$ & $0.51(-0.05 ; 1.06)$ & $-0.10(-0.76 ; 0.57)$ \\
\hline Triglycerides $>1.5 \mathrm{mmol} / \mathrm{l}$ & $-0.07(-0.46 ; 0.31)$ & $0.07(-0.52 ; 0.67)$ & $0.17(-0.23 ; 0.57)$ & $-0.43(-0.91 ; 0.05)$ \\
\hline \multicolumn{5}{|l|}{ Model $3^{c}$} \\
\hline Waist circumference $>102 \mathrm{~cm}$ & $0.08(-0.29 ; 0.45)$ & $0.56(-0.01 ; 1.13)$ & $-0.02(-0.41 ; 0.37)$ & $0.04(-0.42 ; 0.50)$ \\
\hline $\begin{array}{l}\text { Blood pressure } \geq 130 / 85 \text { and/or using anti- } \\
\text { hypertensive medication }\end{array}$ & $0.18(-0.33 ; 0.68)$ & $0.25(-0.53 ; 1.03)$ & $0.16(-0.36 ; 0.69)$ & $-0.15(-0.78 ; 0.47)$ \\
\hline $\begin{array}{l}\text { Blood glucose } \geq 5.6 \mathrm{mmol} / 1 \mathrm{and} / \text { or using anti- } \\
\text { diabetic medication }\end{array}$ & $0.35(-0.03 ; 0.72)$ & $0.06(-0.52 ; 0.65)$ & $0.29(-0.10 ; 0.69)$ & $0.13(-0.34 ; 0.60)$ \\
\hline
\end{tabular}




\begin{tabular}{|c|c|c|c|c|}
\hline HDL-c $<1-03 \mathrm{mmol} / \mathrm{l}$ & $-0.19(-0.72 ; 0.34)$ & $0.22(-0.60 ; 1.04)$ & $0.51(-0.05 ; 1.07)$ & $-0.10(-0.77 ; 0.57)$ \\
\hline Triglycerides $\geq 1.5 \mathrm{mmol} / 1$ & $-0.06(-0.45 ; 0.33)$ & $0.11(-0.49 ; 0.71)$ & $0.18(-0.22 ; 0.59)$ & $-0.42(-0.90 ; 0.06)$ \\
\hline
\end{tabular}

$* p<0.05 * * p<0.01$

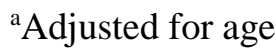

${ }^{\mathrm{b}}$ Adjusted for age, education, BDI score, physical activity, smoking, alcohol consumption, and centre

${ }^{\mathrm{c}}$ Adjusted for age, education, BDI score, physical activity, smoking, alcohol consumption, centre, and co-morbidities

Abbreviations: ROCF, Rey-Osterrieth Complex Figure; CTRM, Camden Topographical Recognition Memory; DSST, Digit Symbol Substitution Test; HDL-c, high-density lipoprotein cholesterol. 
Table $4 \beta$-coefficients (95\% CI) of ordinal logistic regressions of baseline MetS, number of MetS components, individual criteria, and categorical cognitive change in EMAS

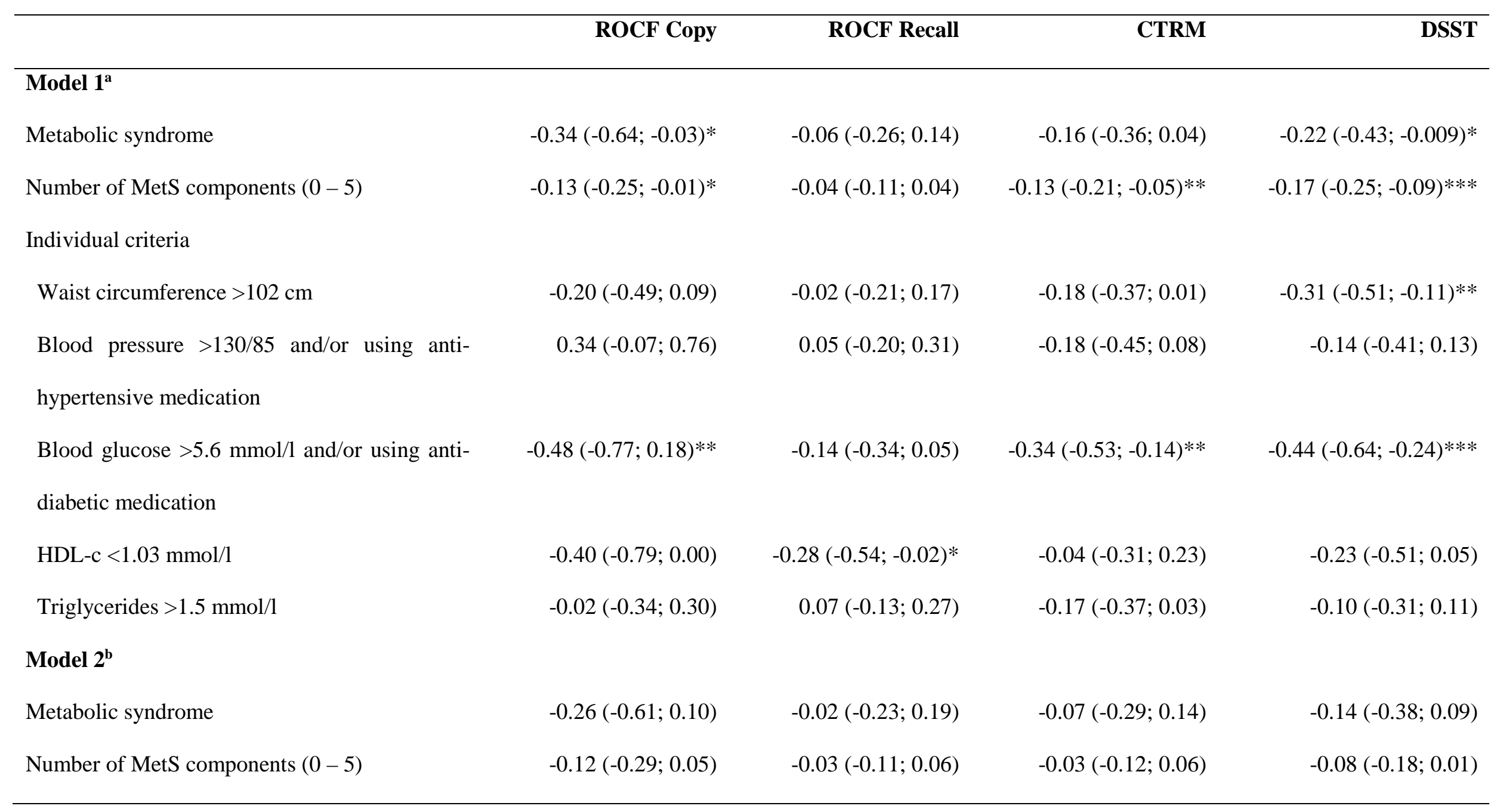




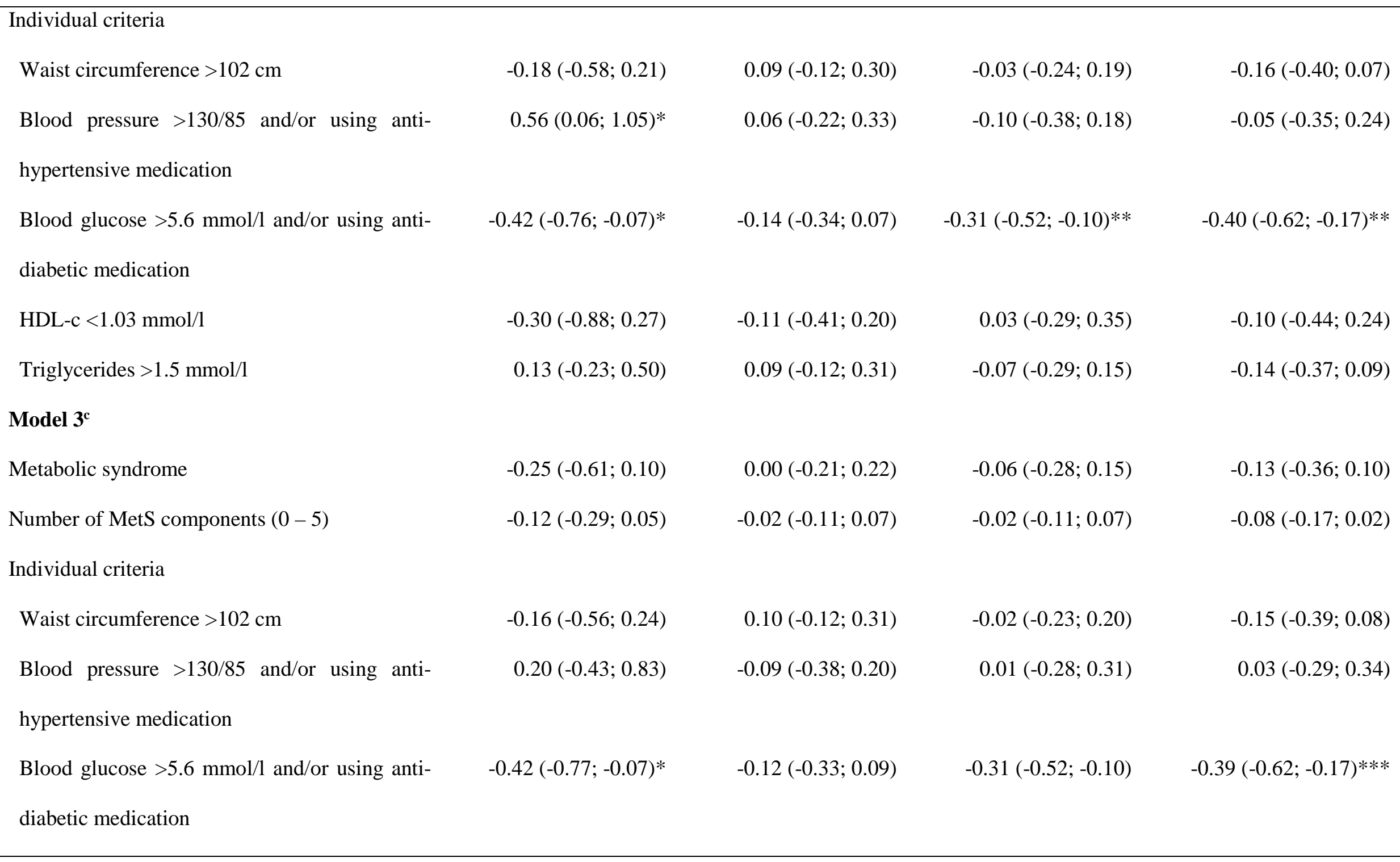




\begin{tabular}{|c|c|c|c|c|}
\hline HDL-c $<1.03 \mathrm{mmol} / \mathrm{l}$ & $-0.28(-0.86 ; 0.29)$ & $-0.09(-0.40 ; 0.21)$ & $0.04(-0.28 ; 0.36)$ & $-0.10(-0.44 ; 0.24)$ \\
\hline Triglycerides >1.5 mmol/l & $0.11(-0.25 ; 0.48)$ & $0.09(-0.12 ; 0.31)$ & $-0.06(-0.28 ; 0.16)$ & $-0.13(-0.36 ; 0.10)$ \\
\hline
\end{tabular}

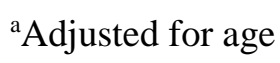

${ }^{\mathrm{b}}$ Adjusted for age, education, BDI score, physical activity, smoking, alcohol consumption, and centre

${ }^{\mathrm{c}}$ Adjusted for age, education, BDI score, physical activity, smoking, alcohol consumption, co-morbidities, and centre

Abbreviations: ROCF, Rey-Osterrieth Complex Figure; CTRM, Camden Topographical Recognition Memory; DSST, Digit Symbol Substitution Test; HDL-c, high-density lipoprotein cholesterol. 